\title{
Prognostic value of the expression of phosphatase and tensin homolog and CD44 in elderly patients with refractory acute myeloid leukemia
}

\author{
XIAO HUANG ${ }^{1}$, DONGYUN LI ${ }^{1}$, TIANTIAN LI ${ }^{1}$, BO ZHAO $^{2}$ and XINYI CHEN ${ }^{1}$ \\ ${ }^{1}$ Department of Oncology and Hematology, Dongzhimen Hospital, Beijing University of Chinese Medicine, \\ Beijing 100700, P.R. China; ${ }^{2}$ Department of Biostatistics, The University of Texas, \\ Houston Health Science Center, Houston, TX 77030, USA
}

Received September 12, 2014; Accepted April 9, 2015

DOI: $10.3892 / 01.2015 .3189$

\begin{abstract}
The leukemic stem cell marker CD44, has been reported to have prognostic significance in hematological malignancies. The present study therefore aimed to evaluate whether the expression levels of CD44 and the associated pathway components are associated with the survival rate of elderly patients with refractory acute myeloid leukemia (AML). A total of 20 elderly patients diagnosed with refractory AML were divided into two groups, following induction chemotherapy: Complete remission $(\mathrm{CR}, \mathrm{n}=9)$ and non-remission (NR. $n=11$ ). Bone marrow biopsy specimens were collected, expression levels of CD44, phosphatase and tensin homolog (PTEN), mammalian target of rapamycin (mTOR) and nuclear factor- $\kappa \mathrm{B}(\mathrm{NF}-\kappa \mathrm{B})$ were analyzed by immunohistochemistry and the captured images were analyzed in a blinded manner using Image Pro Plus software, version 6.0. The overall survival rates (OS) of the patients were then analyzed with log rank, and the correlation between CD44, PTEN, mTOR and NF- $\mathrm{BB}$ expression levels and patients survival rates were statistically analyzed using Pearson's method. Significant differences were observed between the $\mathrm{CR}$ and $\mathrm{NR}$ groups for $\mathrm{PTEN}(\mathrm{P}=0.025)$ and CD44 $(\mathrm{P}=0.020)$ expression levels. Positive CD44 expression was significantly correlated with poor overall survival, with a hazard ratio of $6.281(95 \% \mathrm{CI}, 1.78-22.12$; $\mathrm{P}=0.0042)$. The mean OS was 4.00 months for patients that demonstrated positive CD44 expression, compared with 9.27 months for patients that demonstrated negative CD44 expression. A tendency towards reduced survival rates was also observed in patients negative for PTEN expression, when compared with that of PTEN-positive patients. The mean OS was 4.81 months
\end{abstract}

Correspondence to: Dr Xinyi Chen, Department of Oncology and Hematology, Dongzhimen Hospital, Beijing University of Chinese Medicine, 5 Haiyuncang, Beijing 100700, P.R. China

E-mail:dzmxyzl@gmail.com

Key words: acute myeloid leukemia, CD44, refractory, biomarker, phosphatase and tensin homolog in PTEN-negative patients vs. 8.8 months in PTEN-positive patients, with a hazard ratio of 2.689 (95\% CI, 0.89-8.08; $\mathrm{P}=0.078$ ). Patients that exhibited PTEN-positive and CD44negative expression, survived significantly longer than patients that demonstrated PTEN-negative and CD44-positive expression (mean OS, 9.86 vs 2.67 months; hazard ratio=0.037; 95\% CI, 0.006-0.222, $\mathrm{P}=0.0006)$. The expression levels of $\mathrm{NF}-\kappa \mathrm{B}$ and mTOR were slightly increased in the NR group compared with those of the CR group, although no significant differences were identified. PTEN and CD44 expression levels demonstrated trends towards negative correlation. In conclusion, the expression levels of CD44 and PTEN may be useful markers to predict the prognosis of elderly patients with refractory AML.

\section{Introduction}

Acute myeloid leukemia (AML) is a hematological malignancy with heterogeneous clinical presentations and subtypes. AML has been further classified by the French-American-British Cooperative Group (1-4) and the World Health Organization $(5,6)$ based on the clinical features, and the biological, morphological, immunological and cytogenetic characteristics of the disease. The most common induction therapies for the treatment of adult AML have not changed significantly over the past four decades, and these are chemotherapy or stem cell transplantation (7-9).

Although efforts have been made to develop novel anticancer agents, the overall prognosis for AML has remained poor, particularly amongst older patients. The biological characteristics and clinical features of AML in older adults are different from younger patients, with higher rates of resistance and a poorer response to chemotherapy $(10,11)$. Approximately $70-80 \%$ of younger adults achieve complete remission (CR), with a 5-year-survival rate of $\sim 40-45 \%$ (12). By contrast, CR is achieved in $\sim 40-65 \%$ elderly patients (12) and 5-year-survival rates are $\leq 10 \%$ (13). The most common cause of treatment failure for AML in elderly patients is refractory AML. The underlying mechanism for this resistance of AML to treatment remains unclear (14). Thus, an in-depth understanding of the molecular mechanisms associated with refractory AML is required. 
Table I. Demographics and baseline characteristics of elderly refractory acute myeloid leukemia patients.

A, Demographics

\begin{tabular}{lll}
\hline Characteristic & CR,n $(\%)$ & NR, n $(\%)$ \\
\hline Number & 9 & 11 \\
Gender & & $3(27.3)$ \\
Female & $4(44.4)$ & $8(72.7)$ \\
Male & $5(55.6)$ & 60 \\
Age, years & & $51-73$ \\
Median & 58 & 0.42 \\
Range & $55-77$ & 0.94 \\
\hline
\end{tabular}

$\mathrm{B}$, Baseline laboratory values

\begin{tabular}{lccc}
\hline Laboratory values & Median (range) & Median (range) & P-value \\
\hline White blood cell, $\times 10^{9} / 1$ & $3.6(1.7-8.8)$ & $4.5(1.8-26.1)$ & $80(7-354)$ \\
Platelet count, $x 10^{9} / 1$ & $109(43-226)$ & $82(53-142)$ & 0.57 \\
Hemoglobin, g/1 & $109(81-130)$ & $30(18-94.5)$ & 0.31 \\
Bone marrow blasts, $\%$ & $0.62(0-3)$ & $33(1-80)$ & 0.00 \\
Peripheral blood blasts, $\%$ & $0.00(0.00-2.59)$ & $21.8(8-96)$ & 0.00 \\
Total bilirubin, $\mu$ mol/1 & $10.5(5.5-22)$ & $64.1(38.1-90.4)$ & 0.02 \\
Creatinine, $\mu$ mol $/ 1$ & $55.7(49.3-76)$ & 0.96
\end{tabular}

$\mathrm{CR}$, complete remission group; NR, non-remission group.

Leukemic stem cells (LSCs), which are also termed leukemia-initiating cells, have been reported to be the origin of leukemic cells (15). LSCs serve key functions in the initiation and progression of leukemia and also in relapse or refractory $\mathrm{AML}$, leading to resistance to induction therapies and poor survival outcomes (16). Several LSC biomarkers have been reported to be correlated with refractory or relapse AML and poor prognosis, which may provide instructive information for diagnosis, progression or treatment (17). The LSC surface marker CD44 and components of LSC-associated pathways, including phosphatase and tensin homologue (PTEN), phosphoinositide 3-kinase $(\mathrm{PI} 3 \mathrm{~K}) / \mathrm{Akt} / \mathrm{mammalian}$ target of rapamycin (mTOR) and nuclear factor- $\kappa \mathrm{B}(\mathrm{NF}-\kappa \mathrm{B})$, have been demonstrated to have prognostic value for adult AML patients (18). Previous studies have demonstrated that increased expression levels of CD44 in hematological malignancies is correlated with poor clinical outcomes $(19,20)$. It has also previously been reported that patients in receipt of autologous hematopoietic stem cell transplantations exhibited lower expression levels of CD44 and improved survival outcomes (21). However, the reliability of the correlation between these LSC-associated biomarkers and the prognosis of patients with refractory AML is currently insufficient.

In the present study, the expression levels of CD44, PTEN, mTOR and NF- $\kappa$ B were evaluated in elderly refractory AML patients to determine whether these molecules have prognostic implications and may be potential therapeutic targets for treatment.

\section{Materials and methods}

Patients and tissue samples. Bone marrow (BM) samples were obtained from 20 elderly patients with diagnosed refractory AML $(2,22)$, who were treated at Dongzhimen Hospital (Beijing, China) between December 2011 and April 2013, and possessed complete clinical pathological diagnosis information and the associated follow-up data. The induction chemotherapy drugs that were administered to the patients were Acla, Ara-C, cytoxan, vindesine, epirubicin and VP-16. The bone marrow samples were divided into two groups: CR $(n=9)$ and NR $(n=11)$, following induction chemotherapy treatment. CR was defined as patients with $\leq 5 \%$ leukemic blasts in the BM with signs of normal hematopoiesis and regeneration of normal peripheral-blood cell production (platelets $>1 \times 10^{11} / 1$ without transfusions, neutrophils $\left.>1.5 \times 10^{9} / 1\right)$ and an absence of leukemic cells in the peripheral blood or other locations (22). The refractory AML diagnosis for all patients in the study was confirmed histologically and cytologically. The mean age at diagnosis was $62.05 \pm 8.24$ years and the range was $51-77$ years, 11 patients were male and 8 patients were female. All the patients were followed up until June 2014 or death. The study was approved by the ethics committee of Dongzhimen Hospital and all samples were acquired following informed consent and ethical approval.

Immunohistochemistry. Immunohistochemical (IHC) analysis was performed as previously described (23), with 
Table II. Correlation between PTEN and CD44 expression and baseline characteristics.

\begin{tabular}{|c|c|c|c|c|c|c|}
\hline \multirow[b]{2}{*}{ Characteristic } & \multicolumn{3}{|c|}{ CD44 } & \multicolumn{3}{|c|}{ PTEN } \\
\hline & Positive & Negative & P-value & Positive & Negative & P-value \\
\hline Number, $\mathrm{n}$ & 9 & 11 & & 10 & 10 & \\
\hline \multicolumn{7}{|l|}{ Gender, n (\%) } \\
\hline Female & $4(44.4)$ & $4(33.3)$ & 0.604 & $4(40.0)$ & $7(70.0)$ & 0.64 \\
\hline Male & $5(55.6)$ & $8(66.7)$ & & $6(60.0)$ & $3(30.0)$ & \\
\hline \multicolumn{7}{|l|}{ Age, years } \\
\hline Median & 58 & 60 & 0.400 & 65 & 60 & 0.19 \\
\hline Range & $51-72$ & $55-77$ & & $55-77$ & $51-73$ & \\
\hline \multicolumn{7}{|l|}{$\begin{array}{l}\text { Laboratory values, } \\
\text { median (range) }\end{array}$} \\
\hline WBC, $\times 10^{9} / 1$ & $4.4(1.8-21.0)$ & $3.6(1.7-26.1)$ & 0.856 & $4(1.7-18.7)$ & $3.9(1.8-26.1)$ & 0.76 \\
\hline Platelet count, $\mathrm{x} 10^{9} / 1$ & $74(7-147)$ & $106(43-354)$ & 0.341 & $106(43-226)$ & $95(7-354)$ & 0.93 \\
\hline Hemoglobin, g/l & $82(61-130)$ & $109(53-142)$ & 0.341 & $111.5(71-130)$ & $83(53-142)$ & 0.12 \\
\hline Bone marrow blasts, $\%$ & $22(0-44)$ & $2(0-94.5)$ & 0.526 & $0.81(0-60.5)$ & $26.5(2-94.5)$ & 0.02 \\
\hline Peripheral blood blasts, $\%$ & $4(0-80)$ & $0.15(0-52)$ & 0.312 & $0(0-52)$ & $4(0-80)$ & 0.06 \\
\hline Total bilirubin, $\mu \mathrm{mol} / 1$ & $18.4(8-96)$ & $16.7(5.5-34.5)$ & 0.704 & $10.5(5.5-34.5)$ & $20.3(18-96)$ & 0.14 \\
\hline Creatinine, $\mu \mathrm{mol} / \mathrm{l}$ & $48.8(38.1-74.5)$ & $63.2(52-90.4)$ & 0.056 & $60.5(49.3-76)$ & $52(38-90)$ & 0.35 \\
\hline
\end{tabular}

PTEN, phosphatase and tensin homolog; WBC, white blood cell.

minor modifications. Briefly, sections (4- $\mu \mathrm{m}$ thick) from the paraffin-embedded bone marrow biopsy specimens were collected, deparaffinized, hydrated, heated for antigen retrieval and treated with $1 \%$ bovine albumin serum (Sigma-Aldrich) to prevent non-specific binding. Rabbit anti-human PTEN (1:125; Cell Signaling Technology, Inc., Danvers, MA, USA), rabbit anti-human NF-кB (1:300; Cell Signaling Technology, Inc.), rabbit anti-human mTOR (1:200; Abcam, Cambridge, MA, USA) and rabbit anti-human CD44 (1:100; Abcam) were used as the primary antibodies. Non-specific rabbit anti-human immunoglobulin G (1:200; Abcam) was used as a negative control. Following washing with phosphate-buffered saline (PBS), the sections were incubated with the primary antibodies overnight at $4^{\circ} \mathrm{C}$. Next, the sections were washed three times with PBS and incubated with goat anti-rabbit $\operatorname{IgG}$ secondary antibody $\left(1: 2,000\right.$; Abcam) for $30 \mathrm{~min}$ at $37^{\circ} \mathrm{C}$. The sections were counterstained with $5 \mathrm{~g} / 1$ hematoxylin (Dako North America, Inc., Carpinteria, CA, USA) and $20 \mu \mathrm{l} / \mathrm{ml}$ diaminobenzidine (Dako North America, Inc.) using the Cytomation Envision Plus peroxidase system (Dako North America, Inc.).

Evaluation of immunohistochemical staining. The intensity of the IHC staining was semi-quantitatively scored using a previously described method $(24,25)$. Briefly, according to the positive area that was occupied, expression levels were scored as: - $(0$, negative $),+(1-20 \%$, weak $),++(20-50 \%$, moderate $)$ and $+++(>50 \%$, strong) $(26,27)$. Images of the sections were captured using a microscope (BX51; Olympus Corp., Tokyo, Japan) and a SPOT Imaging Solutions system (Diagnostic Instruments, Inc., Sterling Heights, MI, USA), and analyzed with Image-Pro Plus software, version 6.0 (Media Cybernetics, Inc., Rockville, MD, USA). All the areas were selected from five random visual fields. The mean densitometry of the digital images (magnification, $\mathrm{x}$ 400) were designated as representative staining intensities of PTEN, CD44, mTOR, PI3K and $\mathrm{NF}-\kappa \mathrm{B}$, in order to determine the relative expression levels. All the slides were independently evaluated by two specialized pathologists in a blinded manner, and subjected to statistical analysis.

Statistical analysis. Values are expressed as the mean \pm standard deviation. The statistical evaluations were performed with SPSS software, version 17.0 (SPSS, Inc., Chicago, IL, USA). $\mathrm{P}<0.05$ was considered to indicate a statistically significant difference. Semi-quantitative results were evaluated using the $\chi^{2}$ test. The mean density of protein expressions were measured by Image-Pro Plus and two sample t-tests were used when data were normally distributed, otherwise, non-parametric and Wilcoxon rank sum tests were used for analysis. The correlation was studied using Pearson's coefficient. The cumulative OS analysis was plotted using Kaplan-Meier curves with log rank analysis. The variables were then analyzed by multivariate Cox regression analysis, which was performed to identify independent prognostic survival factors.

\section{Results}

Patient characteristics. A total of 20 bone marrow samples from AML patients were divided into two groups: The CR and NR groups. The median age of the patients was 59 years (range, 51-77 years). The demographics and baseline characteristics of the elderly refractory AML patients are presented in Table I. When comparing the CR and NR groups, the percentage of bone marrow blasts, peripheral blood blasts and the total bili- 

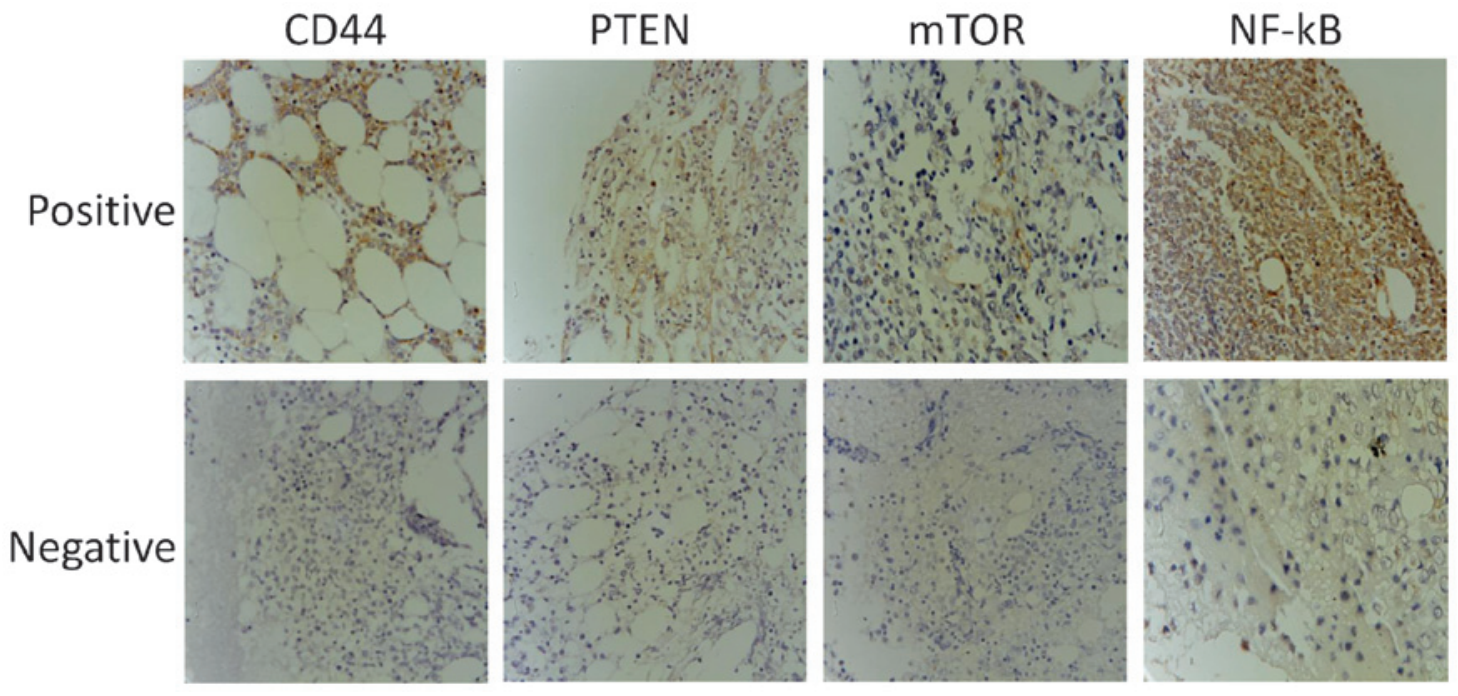

Figure 1. Representative examples of 20 samples of immunohistochemical staining for PTEN, CD44, mTOR and NF-kB in elderly refractory acute myeloid leukemia bone marrow samples. Magnification, x400. PTEN, phosphatase and tensin homolog; mTOR, mammalian target of rapamycin; NF-кB, nuclear factor- $\mathrm{kB}$.

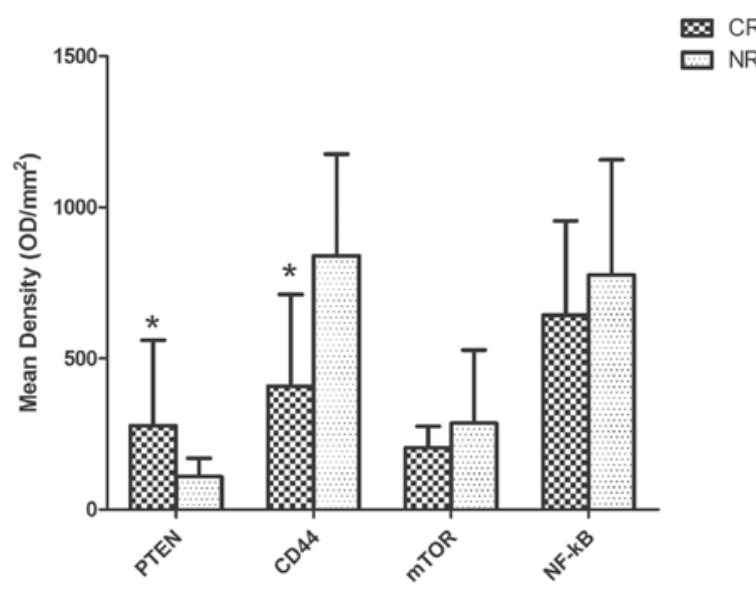

Figure 2. PTEN expression is increased and CD44 expression is decreased in the CR group. Expression of PTEN, CD44, mTOR and NF-kB in the CR $(\mathrm{n}=9)$ and NR $(\mathrm{n}=11)$ groups. Compared with the NR group, bone marrow samples expressed significantly increased PTEN and reduced CD44 levels $\left({ }^{*} \mathrm{P}<0.05\right)$. PTEN, phosphatase and tensin homolog; $\mathrm{CR}$, complete remission group; NR, non-remission group; mTOR, mammalian target of rapamycin;

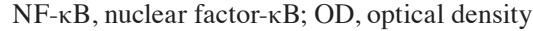

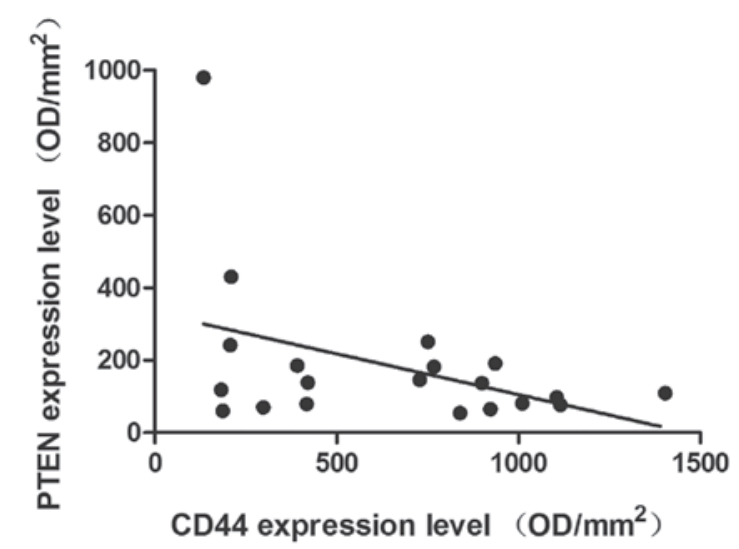

Figure 3. Negative correlation between PTEN expression and CD44 expression $(\mathrm{R}=-0.415 ; \mathrm{P}=0.06)$. PTEN, phosphatase and tensin homolog; OD, optical density. rubin levels were significantly higher in the NR group when compared with the CR group $(\mathrm{P}<0.05$; Table I). These results indicated that patients in the CR and NR groups exhibited different responses to therapy. The percentage of bone marrow blasts was also significantly associated with PTEN expression $(\mathrm{P}=0.016$; Table II); the percentage of bone marrow blasts was significantly higher in PTEN-negative cases when compared with PTEN-positive cases $(\mathrm{P}=0.016)$. It has previously been demonstrated that AML patients with lower bone marrow blast percentages following therapy may exhibit a better prognosis $(28,29)$. Thus, the results of the present study indicate that PTEN expression may also affect the prognosis of AML patients.

PTEN and CD44 are differentially expressed between the two groups. IHC was used to determine the expression levels of PTEN, CD44, mTOR and NF-кB in the patient samples. Representative immunohistochemical stains are presented in Fig. 1. In the CR group the mean densities were as follows: PTEN, 277.32 \pm 283.25 ; CD44, 408.45 \pm 303.47 ; mTOR, 205.72 \pm 242.53 ; and NF- $\mathrm{BB}, 642.66 \pm 312.21$. In the NR group the mean densities were: PTEN, 109.59 \pm 60.85 ; CD44, 840.06 \pm 335.95 ; mTOR, 285.79 \pm 242.53 ; and NF- $\kappa$ B, $776.26 \pm 380.63$, as presented in Fig. 2. The results demonstrated that the PTEN expression levels in the CR group were significantly increased, compared with those of the NR group $(\mathrm{P}=0.025)$, whereas $\mathrm{CD} 44$ was expressed at significantly reduced levels in the CR group compared with that of the NR group ( $\mathrm{P}=0.020$, Fig. 2). By contrast, slightly increased expression levels of NF-KB and mTOR were observed in the NR group compared with those of the CR group, although these differences were not statistically significant ( $P>0.05$, Fig. 2). Furthermore, statistical correlation analysis demonstrated that there was a negative correlation between the expression levels of PTEN and CD44 ( $\mathrm{R}=-0.415$, P=0.069; Fig. 3).

CD44 and PTEN expression levels are associated with patient outcomes. The present study then analyzed whether 
Table III. Univariate and multivariate analysis of overall survival in elderly patients with refractory acute myeloid leukemia.

\begin{tabular}{|c|c|c|c|c|}
\hline \multirow[b]{2}{*}{ Variable } & \multicolumn{2}{|c|}{ Univariate survival analysis } & \multicolumn{2}{|c|}{ Multivariate survival analysis } \\
\hline & Risk ratio $(95 \% \mathrm{CI})$ & P-value & Risk ratio $(95 \% \mathrm{CI})$ & P-value \\
\hline Gender & $1.069(0.391-2.923)$ & 0.896 & $0.502(0.014-17.64)$ & 0.704 \\
\hline Age & $0.999(0.938-1.064)$ & 0.975 & $1.245(0.978-1.584)$ & 0.075 \\
\hline White blood cell & $1.117(1.033-1.208)$ & 0.006 & $1.266(1.017-1.576)$ & 0.035 \\
\hline Platelet count & $0.995(0.987-1.004)$ & 0.303 & $0.945(0.893-0.995)$ & 0.033 \\
\hline Hemoglobin & $0.983(0.961-1.005)$ & 0.136 & $1.036(0.926-1.160)$ & 0.534 \\
\hline Bone marrow blasts & $1.017(0.999-1.036)$ & 0.058 & $1.161(1.003-1.343)$ & 0.046 \\
\hline Peripheral blood blasts & $1.021(1.001-1.041)$ & 0.039 & $0.881(0.783-0.991)$ & 0.035 \\
\hline Total bilirubin & $1.025(0.999-1.052)$ & 0.059 & $0.943(0.875-1.02)$ & 0.126 \\
\hline Creatinine & $0.982(0.942-1.023)$ & 0.392 & $1.112(1.005-1.223)$ & 0.039 \\
\hline PTEN positive & $0.372(0.123-1.117)$ & 0.078 & $0.000(0.913-31.81)$ & 0.056 \\
\hline CD44 positive & $6.281(1.784-22.12)$ & 0.004 & $30.69(0.000-0.208)$ & 0.019 \\
\hline
\end{tabular}

CI, confidence interval.
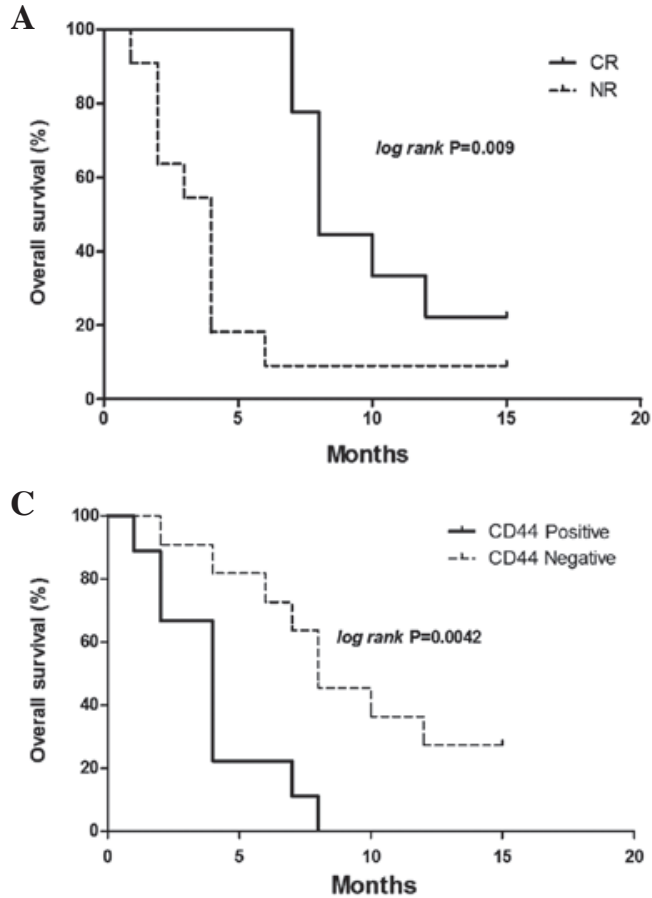

B

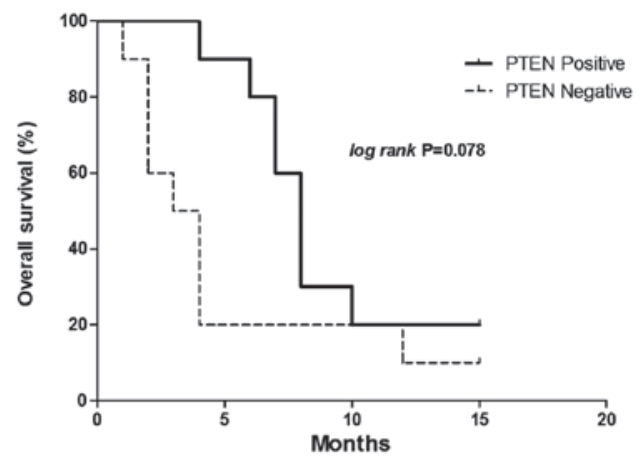

D

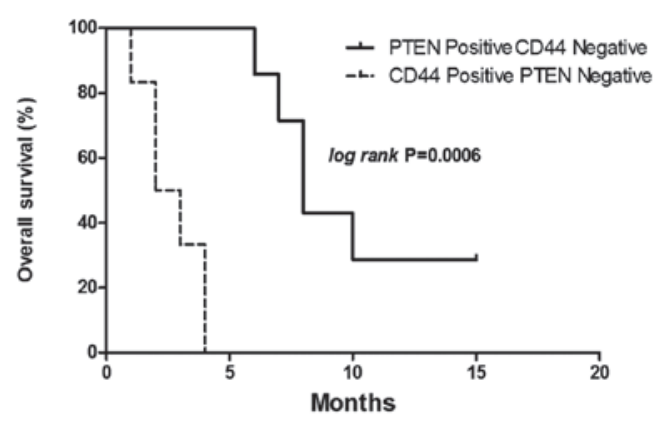

Figure 4. OS of 20 elderly patients with refractory AML. (A) Kaplan-Meier survival curves for the CR $(n=9)$ and NR ( $n=11)$ groups. CR group was associated with significantly increased OS, compared with that of the NR group ( $\mathrm{P}=0.009)$. (B) Kaplan-Meier survival curves for PTEN positive ( $\mathrm{n}=10)$ and negative $(\mathrm{n}=10)$ patients. PTEN positive cases exhibited an increased OS, compared with that of the PTEN negative cases. (C) Kaplan-Meier survival curves for CD44 positive $(n=9)$ and negative $(n=11)$ patients. OS was significantly reduced in CD44 positive cases compared with that of $C D 44$ negative cases $(P=0.0042)$. (D) Cases were divided into a PTEN positive and CD44 negative group $(n=7)$, and a PTEN negative and CD44 positive group ( $n=6)$. Cases determined as either PTEN and CD44 negative or PTEN and CD44 positive were excluded. The difference between the Kaplan-Meier curves was significant ( $\mathrm{P}=0.0006$ on log rank analysis). OS, overall survival; PTEN, phosphatase and tensin homolog; CR, complete remission group; NR, non-remission group.

CD44 and PTEN expression levels were associated with patient outcomes. Kaplan-Meier analysis demonstrated that the mean OS for the CR group was 10.0 months, which was significantly increased compared with that of the NR group (4.27 months; $\mathrm{P}=0.009$ ). CD44 expression levels were significantly associated with the OS in all patients: Patients that were CD44 positive had a mean OS of $\sim 4.0$ months, whereas CD44-negative patients had a mean OS of 9.27 months.
The hazard ratio was 6.281 (95\%CI, 1.78-22.12; $\mathrm{P}=0.0042$; Fig. 4C). Multivariate Cox regression analysis also demonstrated that CD44 was an independent prognostic survival factor for patients with refractory AML ( $\mathrm{P}=0.019$; Table III). There was also a trend towards reduced OS in patients who were PTEN negative when compared with patients who were PTEN positive (mean OS, 4.81 months vs. 8.8 months; hazard ratio, 2.689; 95\% $\mathrm{CI}, 0.89-8.08$; $\mathrm{P}=0.078$; Fig. 4B). The corre- 
lation between PTEN positive and CD44 negative or PTEN negative and CD44 positive patients with OS, was assessed using Kaplan-Meier analysis. Patients that were PTEN positive and CD44 negative had significantly increased OS compared with those that were PTEN negative and CD44 positive. The mean OS for patients that were PTEN negative and CD44 positive was 9.86 months, whereas the mean OS for patients that were PTEN negative and CD44 positive was 2.67 months. The hazard ratio was 0.037 (95\% CI, 0.006-0.222; P=0.0006; Fig. 4D).

\section{Discussion}

In the present study, IHC staining was used to detect the expression of CD44, PTEN, mTOR and NF- $\kappa \mathrm{B}$ in elderly patients with refractory AML, who were divided into $\mathrm{CR}$ and NR groups. It has previously been reported that an increase in CD44 expression contributes to poorer AML prognosis, and CD44 may therefore serve as an adverse prognostic marker (25). The present study indicated that CD44 expression was significantly increased in the NR group compared with that of the CR group. Increased CD44 expression was associated with significantly reduced OS compared with that of CD44 negative patients, which was consistent with the results of a previous report (30). CD44 is a cell surface glycoprotein involved in diverse cellular processes in malignancy, including cell transformation (31), proliferation (31), migration (32) and anti-apoptosis (33). CD44 is also considered to be one of the particular markers that mediate efficient homing and engraftment of LSC in AML (34-36). In addition, CD44 expression is specific to LSC as it is rarely expressed on normal hematopoietic stem cells $(37,38)$. Previous studies have demonstrated that CD44 knockout (CD44 ${ }^{-/}$) mice survived significantly longer and that tumors developed more slowly compared with those of wild-type mice, when injected with murine breast carcinoma cells (30). The expression of CD44 may be associated with adverse prognosis of AML (39), which is consistent with the results obtained in the present study.

In contrast to the expression of CD44, PTEN expression was significantly increased in the CR group compared with that of the NR group. Patients that were PTEN positive tended to have an improved prognosis compared with those that were PTEN negative, although the difference was not statistically significant, possibly due to the relatively small sample size used in the present study. PTEN is a tumor suppressor gene, which is critical for cell proliferation, apoptosis and survival (40-42), and is commonly inactivated in hematological malignancies, resulting in loss of function $(42,43)$. Loss of PTEN function has been demonstrated to be associated with tumor progression (44) and poor prognosis (45), by disturbing the balance of microenvironments, transforming normal stem cells into cancer stem cells (46-48) or promoting the generation of LSCs into unlimited self-renewal (49). The absence of PTEN expression is considered to promote leukemia development and be associated with poor patient outcomes. The results of the present study indicate that further studies with more homogeneous patient samples are required in order to draw conclusions regarding the association between expression of PTEN with the outcome of refractory AML.

It has previously been reported that knockdown of PTEN may upregulate CD44 expression in hepatoma cells and human Huh-7 cells (50). In the present study, when PTEN expression was reduced, OS was significantly improved in the CR group compared with that of the NR group. Furthermore, a significant association between CD44 and PTEN expression was identified. PTEN positive and CD44 negative cases demonstrated a strong correlation with enhanced OS. Analysis of CD44 and PTEN expression was combined and the results demonstrated that there was negative correlation between them. The results of the present study indicate that CD44 may be an adverse prognostic marker, whereas PTEN presents as a favorable biomarker in refractory AML.

PTEN has been shown to negatively regulate the $\mathrm{PI} 3 \mathrm{~K} / \mathrm{Akt} / \mathrm{mTOR}$ and NF- $\mathrm{NB}$ signaling pathways (51), and $\mathrm{NF}-\kappa \mathrm{B}$ and $\mathrm{PI} 3 \mathrm{~K} / \mathrm{Akt} / \mathrm{mTOR}$ signaling is known to be involved in multiple types of cancer (52), promoting carcinogenesis, cancer cell proliferation and apoptosis $(27,53)$. It has previously been reported that the PI3K/Akt/mTOR signaling pathway may be a prognostic marker in gastric cancer. NF- $\kappa \mathrm{B}$ is activated in hematological and solid tumors (54), and has also been demonstrated to be an adverse prognostic factor (55). In children with acute lymphoblastic leukemia, increased expression of $\mathrm{NF}-\kappa \mathrm{B}$ is associated with treatment failure (56). PI3K/Akt/mTOR also activates downstream signaling of Bcr-Abl, which leads to treatment failure and poor clinical outcomes in patients with imatinib (Bcr-Abl inhibitor)-resistant chronic myeloid leukemia (57). The results of the present study demonstrated that there was increased expression of NF- $\kappa \mathrm{B}$ and mTOR in the NR group compared with that of the CR group. However, this increase was not statistically significant, potentially due to the small sample sizes.

In conclusion, the present study provides immunohistochemical evidence that CD44 and PTEN may be used as biomarkers for AML prognosis and for monitoring the response to therapy, which may aid the identification of additional potential strategies to treat refractory disease. The present study used an elderly patient population (>50 years of age) as AML is predominantly diagnosed in elderly patients (1). Notably, older AML patients exhibit a poor prognosis, higher resistance rate and a poorer response to chemotherapy compared with younger patients $(2,3)$. In addition, a CR may only be achieved in 40-65\% of elderly patients(4) and in patients $>55$ years of age, the 5-yearsurvival rate is $<10 \%$ (5). Thus, the elderly population ( $>50$ years of age) were selected for this study with the aim of improving outcomes and the efficacy of treatments for AML. Since CD44, PTEN, mTOR and NF- $\kappa$ B are involved in AML progression and have been demonstrated to affect prognosis, the correlation observed between these biomarkers and prognostic implications in the elderly population may be observed in younger patients, which presents an interesting topic for further research. The present study had certain limitations due to the small sample size, therefore a study with a larger sample size is required to further validate the findings.

\section{Acknowledgements}

The present study was funded by the Specialized Research Fund for the Doctoral Program of Higher Education (grant no. 20100013110008). The authors would like to thank Dr Bingliang Fang (University of Texas MD Anderson Cancer Center, Houston, TX, USA) and Dr Bingbing Dai (University 
of Texas MD Anderson Cancer Center) for language editing and polishing.

\section{References}

1. Bennett JM, Catovsky D, Daniel MT, et al: Proposals for the classification of the acute leukaemias. French-American-British (FAB) co-operative group. Br J Haematol 33: 451-458, 1976.

2. Bennett JM, Catovsky D, Daniel MT, et al: Proposed revised criteria for the classification of acute myeloid leukemia. A report of the French-American-British Cooperative Group. Ann Intern Med 103: 620-625, 1985.

3. Bennett JM, Catovsky D, Daniel MT, et al: Criteria for the diagnosis of acute leukemia of megakaryocyte lineage (M7). A report of the French-American-British Cooperative Group. Ann Intern Med 103: 460-462, 1985.

4. Bennett JM, Catovsky D, Daniel MT, et al: Proposal for the recognition of minimally differentiated acute myeloid leukaemia (AML-MO). Br J Haematol 78: 325-329, 1991.

5. Harris NL, Jaffe ES, Diebold J, et al: The World Health Organization classification of neoplasms of the hematopoietic and lymphoid tissues: Report of the Clinical Advisory Committee meeting - Airlie House, Virginia, November, 1997. Hematol J 1: 53-66, 2000.

6. Vardiman JW, Thiele J, Arber DA, et al: The 2008 revision of the World Health Organization (WHO) classification of myeloid neoplasms and acute leukemia: Rationale and important changes. Blood 114: 937-951, 2009.

7. Yates JW, Wallace HJ Jr, Ellison RR and Holland JF: Cytosine arabinoside (NSC-63878) and daunorubicin (NSC-83142) therapy in acute nonlymphocytic leukemia. Cancer Chemother Rep 57: 485-488, 1973.

8. Rai KR, Holland JF, Glidewell OJ, et al: Treatment of acute myelocytic leukemia: A study by cancer and leukemia group B Blood 58: 1203-1212, 1981.

9. O'Donnell MR, Abboud CN, Altman J, et al: Acute myeloid leukemia. J Natl Compr Canc Netw 10: 984-1021, 2012.

10. Swords R and Santini V: In elderly patients with AML, which patients should be considered fit or unfit for standard induction therapy? Hematology Am Soc Hematol Educ Program 2012: 74-75, 2012.

11. Yanada M and Naoe T: Acute myeloid leukemia in older adults. Int J Hematol 96: 186-193, 2012.

12. Burnett A, Wetzler M and Löwenberg B: Therapeutic advances in acute myeloid leukemia. J Clin Oncol 29: 487-494, 2011

13. Dombret H, Raffoux E and Gardin C: Acute myeloid leukemia in the elderly. Semin Oncol 35: 430-438, 2008.

14. Krug U, Büchner T, Berdel WE and Müller-Tidow C: The treatment of elderly patients with acute myeloid leukemia. Dtsch Arztebl Int 108: 863-870, 2011.

15. Jordan CT: The leukemic stem cell. Best Pract Res Clin Haematol 20: 13-18, 2007.

16. She M, Niu X, Chen X, et al: Resistance of leukemic stem-like cells in AML cell line KG1a to natural killer cell-mediated cytotoxicity. Cancer Lett 318: 173-179, 2012.

17. Ardekani AM, Akhondi MM and Sadeghi MR: Application of genomic and proteomic technologies to early detection of cancer. Arch Iran Med 11: 427-434, 2008.

18. Biomarkers Definitions Working Group: Biomarkers and surrogate endpoints: Preferred definitions and conceptual framework. Clin Pharmacol Ther 69: 89-95, 2001.

19. Eisterer W, Bechter O, Hilbe W, et al: CD44 isoforms are differentially regulated in plasma cell dyscrasias and CD44v9 represents a new independent prognostic parameter in multiple myeloma. Leuk Res 25: 1051-1057, 2001.

20. Niitsu N and Iijima K: High serum soluble CD44 is correlated with a poor outcome of aggressive non-Hodgkin's lymphoma. Leuk Res 26: 241-248, 2002.

21. Krause DS, Spitzer TR and Stowell CP: The concentration of CD44 is increased in hematopoietic stem cell grafts of patients with acute myeloid leukemia, plasma cell myeloma, and non-Hodgkin's lymphoma. Arch Pathol Lab Med 134: 1033-1038, 2010.

22. Mi Y and Bian S: Acute leukemia. In: Standardization of Hematologic Disease Diagnosis and the Therapeutic Effect. Zhinan Z and Ti S (eds). Vol 1. 3rd edition. Science Press, Beijing, China, pp131-134, 2007.

23. Wang $\mathrm{Z}$, Zheng $\mathrm{T}$, Wu $\mathrm{Q}$, Wang $\mathrm{J}$, Wu $\mathrm{C}$ and Wang $\mathrm{J}$ : Immunohistochemical analysis of the mTOR pathway in intrahepatic cholangiocarcinoma. Neoplasma 59: 137-141, 2012.
24. Hu J, Liu YL, Piao SL, et al: Expression patterns of USP22 and potential targets BMI-1, PTEN, p-AKT in non-small-cell lung cancer. Lung Cancer 77: 593-599, 2012.

25. Cao L, Hu X, Zhang J, Liang P and Zhang Y: CD44 U(+) CD324(-) expression and prognosis in gastric cancer patients. J Surg Oncol 110: 727-733, 2014

26. Opitz I, Soltermann A, Abaecherli M, et al: PTEN expression is a strong predictor of survival in mesothelioma patients. Eur J Cardiothorac Surg 33: 502-506, 2008.

27. Tapia O, Riquelme I, Leal P, et al: The PI3K/AKT/mTOR pathway is activated in gastric cancer with potential prognostic and predictive significance. Virchows Arch 465: 25-33, 2014.

28. Sebban C, Browman GP, Lepage E and Fière D: Prognostic value of early response to chemotherapy assessed by the day 15 bone marrow aspiration in adult acute lymphoblastic leukemia: A prospective analysis of 437 cases and its application for designing induction chemotherapy trials. Leuk Res 19: 861-868, 1995.

29. Cortes J, Fayad L, O'Brien S, Keating M and Kantarjian H: Persistence of peripheral blood and bone marrow blasts during remission induction in adult acute lymphoblastic leukemia confers a poor prognosis depending on treatment intensity. Clin Cancer Res 5: 2491-2497, 1999.

30. Fitzgerald KA, Bowie AG, Skeffington BS and O'Neill LA: Ras, protein kinase $\mathrm{C}$ zeta, and I kappa B kinases 1 and 2 are downstream effectors of CD44 during the activation of NF-kappa B by hyaluronic acid fragments in T-24 carcinoma cells. J Immunol 164: 2053-2063, 2000.

31. Bourguignon LY, Zhu H, Shao L, Zhu D and Chen YW: Rho-kinase (ROK) promotes CD44v(3,8-10)-ankyrin interaction and tumor cell migration in metastatic breast cancer cells. Cell Motil Cytoskeleton 43: 269-287, 1999.

32. Lin YH and Yang-Yen HF: The osteopontin-CD44 survival signal involves activation of the phosphatidylinositol 3-kinase/Akt signaling pathway. J Biol Chem 276: 46024-46030, 2001.

33. Dick JE, Bhatia M, Gan O, Kapp U and Wang JC: Assay of human stem cells by repopulation of NOD/SCID mice. Stem Cells 15: 199-207, 1997.

34. Krause DS and Van Etten RA: Right on target: Eradicating leukemic stem cells. Trends Mol Med 13: 470-481, 2007.

35. Oelschlaegel U, Bornhauser M, Boxberger S, et al: Kinetics of CXCR-4 and adhesion molecule expression during autologous stem cell mobilisation with G-CSF plus AMD3100 in patients with multiple myeloma. Ann Hematol 86: 569-573, 2007.

36. van Rhenen A, Moshaver B, Kelder A, et al: Aberrant marker expression patterns on the CD34+CD38- stem cell compartment in acute myeloid leukemia allows to distinguish the malignant from the normal stem cell compartment both at diagnosis and in remission. Leukemia 21: 1700-1707, 2007.

37. Buzzai M and Licht JD: New molecular concepts and targets in acute myeloid leukemia. Curr Opin Hematol 15: 82-87, 2008.

38. Spaeth EL, Labaff AM, Toole BP, et al: Mesenchymal CD44 expression contributes to the acquisition of an activated fibroblast phenotype via TWIST activation in the tumor microenvironment. Cancer Res 73: 5347-5359, 2013.

39. Lane SW, Scadden DT and Gilliland DG: The leukemic stem cell niche: Current concepts and therapeutic opportunities. Blood 114: 1150-1157, 2009.

40. Maehama $T$ and Dixon JE: The tumor suppressor, PTEN/MMAC1, dephosphorylates the lipid second messenger, phosphatidylinositol 3,4,5-trisphosphate. J Biol Chem 273: 13375-13378, 1998.

41. Stiles B, Groszer M, Wang S, Jiao J and Wu H: PTENless means more. Dev Biol 273: 175-184, 2004.

42. Dahia PL, Aguiar RC, Alberta J, et al: PTEN is inversely correlated with the cell survival factor $\mathrm{Akt} / \mathrm{PKB}$ and is inactivated via multiple mechanisms in haematological malignancies. Hum Mol Genet 8: 185-193, 1999.

43. Cheong JW, Eom JI, Maeng HY, et al: Phosphatase and tensin homologue phosphorylation in the C-terminal regulatory domain is frequently observed in acute myeloid leukaemia and associated with poor clinical outcome. Br J Haematol 122: 454-456, 2003.

44. Squarize CH, Castilho RM, Abrahao AC, et al: PTEN deficiency contributes to the development and progression of head and neck cancer. Neoplasia 15: 461-471, 2013.

45. da Costa AA, D'Almeida Costa F, Ribeiro AR, et al: Low PTEN expression is associated with worse overall survival in head and neck squamous cell carcinoma patients treated with chemotherapy and cetuximab. Int J Clin Oncol: May 27, 2014 (Epub ahead of print) 
46. Hede K: PTEN takes center stage in cancer stem cell research, works as tumor suppressor. J Natl Cancer Inst 98: 808-809, 2006.

47. He XC, Yin T, Grindley JC, et al: PTEN-deficient intestinal stem cells initiate intestinal polyposis. Nat Genet 39: 189-198, 2007.

48. Yilmaz OH, Valdez R, Theisen BK, et al: Pten dependence distinguishes haematopoietic stem cells from leukaemia-initiating cells. Nature 441: 475-482, 2006.

49. Tesio M, Oser GM, Baccelli I, et al: Pten loss in the bone marrow leads to G-CSF-mediated HSC mobilization. J Exp Med 210: 2337-2349, 2013

50. Chu TH, Chan HH, Kuo HM, et al: Celecoxib suppresses hepatoma stemness and progression by up-regulating PTEN. Oncotarget 5: 1475-1490, 2014.

51. Salminen A and Kaarniranta K: Insulin/IGF-1 paradox of aging: Regulation via AKT/IKK/NF-kappaB signaling. Cell Signal 22: 573-577, 2010.

52. Dubrovska A, Kim S, Salamone RJ, et al: The role of PTEN/Akt/PI3K signaling in the maintenance and viability of prostate cancer stem-like cell populations. Proc Natl Acad Sci USA 106: 268-273, 2009.
53. Kim HJ, Hawke N and Baldwin AS: NF-kappaB and IKK as therapeutic targets in cancer. Cell Death Differ 13: 738-747, 2006.

54. Lee CH, Jeon YT, Kim SH and Song YS: NF-kappaB as a potential molecular target for cancer therapy. Biofactors 29: 19-35, 2007.

55. Breccia $\mathrm{M}$ and Alimena $\mathrm{G}: \mathrm{NF}-\mathrm{\kappa B}$ as a potential therapeutic target in myelodysplastic syndromes and acute myeloid leukemia. Expert Opin Ther Targets 14: 1157-1176, 2010.

56. Kamieńska E, Ociepa T, Wysocki M, et al: Activation of NF-кB in leukemic cells in response to initial prednisone therapy in children with acute lymphoblastic leukaemia: Relation to other prognostic factors. Pol J Pathol 62: 5-11, 2011.

57. Lounnas N, Frelin C, Gonthier N, et al: NF-kappaB inhibition triggers death of imatinib-sensitive and imatinib-resistant chronic myeloid leukemia cells including T315I Bcr-Abl mutants. Int J Cancer 125: 308-317, 2009. 